\title{
Liver Stiffness Measurement Can Reflect the Active Liver Necroinflammation in Population with Chronic Liver Disease: A Real-world Evidence Study
}

\author{
Leijie Wang ${ }^{\# 1}$, Mingyu Zhü2 ${ }^{\# 2}$ Lihua Cao ${ }^{\# 3}$, Mingjie Yao ${ }^{\# 4}$, Yiwei Lu ${ }^{5}$, Xiajie Wen ${ }^{1}$, Ying Zhang ${ }^{6}$, \\ Jing Ning ${ }^{1}$, Huiling Long ${ }^{7}$, Yueyong Zhu ${ }^{8}$, Guoxin $\mathrm{Hu}^{9}$, Shuangsuo Dang ${ }^{10}$, Qingchun Fu ${ }^{11}$, \\ Liang Chen ${ }^{12}$, Xinxin Zhang ${ }^{2}$, Jingmin Zhao ${ }^{13}$, Zhiliang Gao ${ }^{7}$, Yuemin Nan*6 \\ and Fengmin Lu*1,14
}

\begin{abstract}
${ }^{1}$ Department of Microbiology \& Infectious Disease Center, School of Basic Medical Sciences, Peking University Health Science Center, Beijing, China; ${ }^{2}$ Department of Infectious Diseases, Rui Jin Hospital, Shanghai Jiao Tong University School of Medicine, Shanghai, China; ${ }^{3}$ Liver Disease Center, Qinhuangdao Third Hospital, Qinhuangdao, Hebei, China; ${ }^{4}$ Department of Anatomy and Embryology, School of Basic Medical Sciences, Peking University Health Science Center, Beijing, China; ${ }^{5}$ Eshelman School of Pharmacy, University of North Carolina, Chapel Hill, NC, USA; ${ }^{6}$ Department of Traditional and Western Medical Hepatology, The Third Hospital of Hebei Medical University, Shijiazhuang, Hebei, China; ${ }^{7}$ Department of Infectious Diseases, The Third Affiliated Hospital of Sun Yat-Sen University, Guangzhou, Guangdong, China; ${ }^{8}$ Liver Research Center, First Affiliated Hospital of Fujian Medical University, Fuzhou, Fujian, China; ${ }^{9}$ Department of Infectious Diseases, Peking University Shenzhen Hospital, Shenzhen, Guangdong, China; ${ }^{10}$ Department of Infectious Diseases, Second Affiliated Hospital of Medical College of Xi'an Jiaotong University, Xi'an, Shaanxi, China; ${ }^{11}$ Shanghai Liver Diseases Research Center, 85th Hospital, Nanjing Military Command, Shanghai, China; ${ }^{12}$ Department of Liver Disease, Shanghai Public Health Clinical Center, Fudan University, Shanghai, China;

${ }^{13}$ Department of Pathology and Hepatology, The Fifth Medical Center of PLA General Hospital, Beijing, China; ${ }^{14}$ Intervention and Cell Therapy Center, Peking University Shenzhen Hospital, Shenzhen, Guangdong, China
\end{abstract}

\begin{abstract}
Background and Aims: Non-invasive evaluation of liver necroinflammation in patients with chronic liver disease is an unmet need in clinical practice. The diagnostic accuracy of transient elastography-based liver stiffness measurement (LSM) for liver fibrosis could be affected by liver necroinflammation, the latter of which could intensify stiffness of the liver. Such results have prompted us to explore the diagnosis potential of LSM for liver inflammation. Methods: Three cross-sectional cohorts of liver biopsy-proven chronic liver disease patients were enrolled, including 1417 chronic hepatitis $B$ (CHB) patients from 10 different medical centers, 106 non-alcoholic steatohepatitis patients, and 143 patients with autoimmune-related liver diseases. Another longitudinal cohort of 14 entecavir treatment patients was also included. The receiver operating characteristic (ROC) curve was employed to explore the diagnostic value of LSM. Results: In CHB patients,
\end{abstract}

Keywords: Liver stiffness measurement; Liver necroinflammatory grade; Alanine aminotransferase; Chronic liver disease.

Abbreviations: AUROC, area under the receiver operating characteristic curve; $\mathrm{AIH}$, autoimmune hepatitis; CHB, chronic hepatitis B; LSM, liver stiffness measurement; $\mathrm{NASH}$, non-alcoholic steatohepatitis; $\mathrm{PBC}$, primary biliary cholangitis; ROC, receiver operating characteristic curve; TE, transient electrograph.

Received: 28 August 2019; Revised: 1 November 2019; Accepted: 5 November 2019

\#These authors contributed equally to this study.

* Correspondence to: Yuemin Nan,. Department of Traditional and Western Medical Hepatology, The Third Hospital of Hebei Medical University, 139 Ziqiang Road, Shijiazhuang, Hebei 050017, China. E-mail: nanyuemin@163.com; Fengmin Lu, Department of Microbiology \& Infectious Disease Center, School of Basic Medical Sciences, Peking University Health Science Center, 38 Xueyuan Road, Beijing 100191, China. Tel: +86-10-82805136, E-mail: lu.fengmin@hsc. pku.edu.cn
LSM value ascended with the increased severity of liver necroinflammation in patients with the same fibrosis stage. Such positive correlation between LSM and liver necroinflammation was also found in non-alcoholic steatohepatitis and autoimmune-related liver diseases populations. Furthermore, the ROC curve exhibited that LSM could identify moderate and severe inflammation in CHB patients (area under the ROC curve as 0.779 and 0.838 ) and in non-alcoholic steatohepatitis patients (area under the ROC curve as 0.826 and 0.871), respectively. Such moderate diagnostic value was also found in autoimmune-related liver diseases patients. In addition, in the longitudinal entecavir treated CHB cohort, a decline of LSM values was observed in parallel with the control of inflammatory activity in liver. Conclusions: Our study implicates a diagnostic potential of LSM to evaluate the severity of liver necroinflammation in chronic liver disease patients.

Citation of this article: Wang $L$, Zhu M, Cao L, Yao M, Lu Y, Wen $X$, et al. Liver stiffness measurement can reflect the active liver necroinflammation in population with chronic liver disease: A real-world evidence study. J Clin Transl Hepatol 2019;7(4):313-321. doi: 10.14218/JCTH.2019.00040.

\section{Introduction}

Chronic liver diseases, including viral hepatitis and non-viral hepatitis, are a heavy health burden worldwide. There are approximately 2 million deaths caused by liver disease each year, with 1.16 million due to liver cirrhosis and 0.79 million due to primary liver cancer. ${ }^{1}$ Chronic hepatitis $B(C H B)$ is a major cause of liver cirrhosis and hepatocellular carcinoma. Prompt anti-viral therapy is able to slow down CHB progression. ${ }^{2,3}$ In 
Wang L. et al: LSM predicts liver necroinflammation in CLD

addition, non-alcoholic fatty liver disease, a major causative factor of non-viral hepatitis and cause of $14.1 \%$ of hepatocellular carcinoma cases, has gotten much attention in recent years. ${ }^{4,5}$ Non-alcoholic fatty liver disease can be divided into non-alcoholic fatty liver and non-alcoholic steatohepatitis (NASH), based on histologic findings. ${ }^{6}$ In those NASH patients, active liver inflammation (A2-A3) is an independent predictive factor for non-alcoholic fatty liver disease-related extrahepatic complications. ${ }^{7}$ The prognosis and treatment strategy of chronic liver diseases depends strongly on the degree of liver inflammatory activity. Thus, to slow down chronic liver disease progression and to prevent the occurrence of end-stage liver disease, it is necessary to differentiate those chronic liver disease patients with active liver necroinflammation for timely intervention.

Non-invasive methods, including surrogate serum markers and imaging techniques, offer cost effective alternatives to liver biopsy. Alanine aminotransferase and aspartate aminotransferase, both indicators of liver injury, are widely used. But there are limitations in their accuracy for inflammation in $\mathrm{CHB}$, and they are almost completely unusable in NASH patients. ${ }^{8,9}$ In NASH patients, studies have shown that CK-18, a recently emerging serum biomarker for non-alcoholic fatty liver disease-related inflammation, only exhibits limited diagnostic value. Thus, the diagnosis of active necroinflammatory liver damage in non-alcoholic fatty liver disease is still solely dependent on liver biopsies. ${ }^{10}$ Currently, there is still lack of non-invasive method to diagnose and grade liver necroinflammation in clinical practice.

Liver stiffness measurement (LSM) performed by transient electrograph (TE) has been becoming an increasingly commonly used non-invasive method for the diagnosis of liver cirrhosis. It has been widely noticed that the diagnostic accuracy of LSM for fibrosis/cirrhosis can be affected by several factors including liver necroinflammation, ${ }^{11}$ which was considered to weaken LSM's diagnostic accuracy for liver fibrosis/cirrhosis. ${ }^{12,13}$ In several recently published studies, sequential measurements of LSM revealed that LSM decreases after initiating anti-viral therapy in patients with $\mathrm{CHB}$. One suggested interpretation is that an early steep decline of liver stiffness may predict histological reversal of fibrosis. ${ }^{14}$ However, the 'China HepB-Related Fibrosis Assessment Research Group' argues that a decline in liver stiffness cannot indicate fibrosis regression in patients with $\mathrm{CHB}^{15}$ In this prospective study, the authors noticed that a greater decline of LSM was correlated with a change in HAI scores $(r=0.395, p=0.000)$ but only mildly correlated with change in the Ishak fibrosis stage $(r=0.156, p=0.036)$. We wondered whether such inconsistencies may be caused by a difference in liver inflammation stages at baseline and the therapy received between the two groups. Since the LSM value is closely associated with active liver inflammation, an alternative interpretation of an elevated LSM is that it may reflect the inflammatory grade of a chronic liver disease patient. From this perspective, it is reasonable to postulate that LSM may possesses the diagnostic potential for active liver inflammation.

Thus, we investigated in this study if LSM could be used as a non-invasive method to evaluate liver inflammation in chronic liver disease patients with different etiological backgrounds, including CHB patients, NASH patients, and patients with autoimmune-related liver diseases. Notably, the evaluation of LSM's diagnostic value for liver necroinflammation in CHB cohorts was implemented at multiple centers.

\section{Methods}

\section{Patients and ethical consent}

In this retrospective study, three cross-sectional cohorts were used:

CHB cohorts: In order to further examine the LSM's value in identifying active liver necroinflammation, liver biopsyconfirmed CHB patients were enrolled. $\mathrm{CHB}$ patients were recruited from the following 10 centers: The Fifth Medical Center of PLA General Hospital, The Third Hospital of Hebei Medical University, Rui Jin Hospital, The Third Affiliated Hospital Sun Yat-Sen University, The Third Hospital of Qinhuangdao, Shanghai Public Health Clinical Center, Peking University Shenzhen Hospital, The First Affiliated Hospital of Fujian Medical University, $85^{\text {th }}$ Hospital, and the Second Affiliated Hospital of Medical College of Xi'an Jiaotong University.

All hospitals followed the same criteria for the patient inclusion and exclusion. Inclusion criteria: 1) diagnosed with $\mathrm{CHB}$, defined as the persistent presence of hepatitis $\mathrm{B}$ surface antigen for at least 6 months, with signs of chronic hepatitis; ${ }^{16}$ 2) LSM performed within 1 week of when the liver biopsy was performed; and 3) clinical index data collected on the same day when the liver biopsy was applied. Exclusion criteria: 1 ) patients who were treated with anti-viral treatment within the past 6 months; or 2) evidence of hepatocellular carcinoma.

NASH cohort: From 2011 to 2016, liver biopsy-confirmed NASH patients were enrolled from The Third Hospital of Hebei Medical University. The clinical criteria of NASH were as follows: NASH was diagnosed based on liver biopsy after exclusion of, 1) concomitant steatosis-inducing drugs, 2) excessive alcohol consumption ( $>210 \mathrm{~g} /$ week in men or $>140 \mathrm{~g} /$ week in women), CHB or chronic hepatitis C viral infection, 3) histological evidence of other concomitant chronic liver disease, ${ }^{17}$ and 4) evidence of hepatocellular carcinoma. In addition, the LSMs were performed within 1 week of when the liver biopsy was performed, and the blood test was conducted on the same day as the liver biopsy.

Autoimmune-related liver diseases cohort: Patients in this cohort partly belonged to a previously study, ${ }^{18}$ which included 66 autoimmune hepatitis (AIH) patients and 77 primary biliary cholangitis (PBC) patients.

Longitudinal cohort: This cohort was comprised of 14 CHB patients enrolled from The Third Hospital of Qinhuangdao, all of who underwent entecavir treatment $(0.5 \mathrm{mg} / \mathrm{d}$ for 78 weeks) and had liver biopsy at baseline and at 78 weeks post-treatment initiation. LSM and blood tests were conducted at 13, 26, 52 and 78 weeks. Part of the patients' information have been described in the report from the China HepB-Related Fibrosis Assessment Research Group, which was published during our manuscript submission. ${ }^{15}$

All sample tests in this study were performed in qualified laboratories of each center, and the results were comparable. Ethical consents were obtained by the Central Ethics Committee of The Third Hospital of Hebei Medical University, the Ethics Committee of Rui Jin Hospital, and the Medical Ethics Committee of The Third Affiliated Hospital Sun Yat-Sen University. The diagnosis and treatment of the patients followed the Guideline of Prevention and Treatment for Chronic Hepatitis B. ${ }^{19}$ Informed consent was obtained from all patients in this study and parents' consent was obtained if the patients were under 18 . 


\section{Liver biopsy}

The necroinflammatory grade was evaluated according to the Scheuer system ${ }^{20}$ in CHB patients and the Brunt system ${ }^{21}$ in NASH patients respectively. G2 or $>\mathrm{G} 2$ was defined as moderate liver necroinflammation and $\mathrm{G} 3$ was defined as severe liver necroinflammation in the $\mathrm{CHB}$ patients and $\mathrm{NASH}$ patients.

\section{LSM performance}

LSM was performed with FibroTouch (HISKY Medical Technologies Co., Ltd., Beijing, China) in Rui Jin Hospital and The Third Affiliated Hospital Sun Yat-Sen University, or Fibroscan device (Echosens, Pairs, France) in other medical centers within 1 week before liver biopsy. LSM was deter-mined in the right hepatic lobe through the intercostals space (at least 2 hours after the last meal) with the patient in the supine position and the right arm in maximum abduction. Only valid measurements (success rate of more than $60 \%$ and interquartile range/median ratio of $<0.3$ ) were included. The results were expressed in $\mathrm{kPa}$ corresponding to the median of 10 determinations.

\section{Statistical analysis}

Categorical data were presented as a number and percentage, and the results were analyzed by chi-squared test. Covariance analysis was used to adjust the effect of other variables. The data was analyzed with IBM SPSS statistical software version 22.0 (IBM Corp., New York, NY, USA) and performed with GraphPad Prism version 5.0 (GraphPad Software Inc., La Jolla, CA, USA). The Spearman's rank correlation test and its coefficient (rho) was used to describe the association between two variables. The area under the ROC curve (AUROC), sensitivity, specificity, positive likelihood ratios, negative likelihood ratios, and diagnostic odds ratios were used to evaluate the diagnostic value. We defined AUROC $>0.9$ as excellent, $0.9-0.8$ as good, and 0.7-0.8 as fair. $^{22}$ The positive likelihood ratio is the ratio between true positive and false positive, while the negative likelihood ratio is the ratio between false negative and true negative. The positive likelihood ratios $>10$ or negative likelihood ratios $<0.1$ indicated the high diagnostic value of the index. The significance of differences between the groups was judged by the Z-test. The ROC curve was made by using Medcalc (15.6.1). All tests of significance were two-tailed and $p$ values $<0.05$ were considered statistically significant.

The data from the five independent cohorts were analyzed by using RevMan 5.3, a software usually used for metaanalysis of multicenter data. In summary, ROCs were plotted.

\section{Results}

\section{Clinical characteristics of patients}

As shown in Table 1, a total of 1417 CHB patients from 10 different medical centers and $106 \mathrm{NASH}$ patients from The Fifth Medical Center of PLA General Hospital were enrolled. The $\mathrm{CHB}$ patients were grouped into five independent cohorts: cohort A from The Fifth Medical Center of PLA General Hospital ( $n=269$ ); cohort B from The Third Hospital of Hebei Medical University $(n=253)$; cohort C from Rui Jin Hospital $(n=549)$; cohort D from The Third Affiliated Hospital Sun Yat-Sen
Table 1. Clinical characteristic of patients

\begin{tabular}{lll}
\hline Variable & CHB & NASH \\
\hline Number & 1417 & 106 \\
Sex, male/female & $961 / 456$ & $58 / 48$ \\
Age (years) & $37(30,46)$ & $40(26,50)$ \\
PLT $\left(10^{9} / \mathrm{L}\right)$ & $178(142,218)$ & $213(180,250)$ \\
ALT $(\mathrm{U} / \mathrm{L})$ & $42(28,75)$ & $76(40,119)$ \\
AST $(\mathrm{U} / \mathrm{L})$ & $33(25,51)$ & $44(30,65)$ \\
ALP $(\mathrm{IU} / \mathrm{L})$ & $72(59,90)$ & $95(74,120)$ \\
GGT $(\mathrm{IU} / \mathrm{L})$ & $28(18,49)$ & $58(41,93)$ \\
LSM $(\mathrm{kPa})$ & $8(6,11.9)$ & $6.9(5.4,9.2)$ \\
Liver & $784 / 454 / 179$ & $70 / 30 / 6$ \\
necroinflammation, & & \\
$0-1 / 2 / 3$ & & $64 / 20 / 13 / 9$ \\
Fibrosis stage, & $656 / 360 / 190 /$ & \\
$0-1 / 2 / 3 / 4$ & 211 & \\
\hline
\end{tabular}

Abbreviations: ALP, alkaline phosphatase; ALT, alanine aminotransferase; AST, aspartate aminotransferase; CHB, chronic hepatitis B; GGT, gamma-glutamyl transpeptidase; LSM, liver stiffness measurement; NASH, non-alcoholic steatohepatitis; PLT, platelet.

University ( $n=207$ ); and cohort E from the remaining six medical centers $(n=139)$, with 28 from The Third Hospital of Qinhuangdao, 25 from Shanghai Public Health Clinical Center, 33 from Peking University Shenzhen Hospital, 23 from The First Affiliated Hospital of Fujian Medical University, 26 from The $85^{\text {th }}$ Hospital, and 4 from Xi'an Jiaotong University (Supplementary Fig. 1 for detail). In addition, $14 \mathrm{CHB}$ patients were accepted as part of a 78-week treatment longitudinal cohort. The clinical characteristics of these cross-sectional cohorts are shown in Table 1 . The difference between patients $\mathrm{G} 0-1$ and the patients $\geq \mathrm{G} 2$ are shown in Supplementary Table 1. As expected, patients with a higher liver necroinflammatory grade had a higher level of alanine aminotransferase, aspartate aminotransferase, alkaline phosphatase, gamma-glutamyl transpeptidase and LSM, while sex and age had no difference between the two groups. The clinical characteristic of AIH and PBC was partly described in previous study. ${ }^{18}$

\section{LSM value correlated well with liver necroinflammatory grade in a variety of different chronic liver diseases}

First, to validate the quality of our study cohorts, the correlation of LSM to the scores of liver fibrosis/cirrhosis was analyzed. Consistent with previous studies, LSM exhibited a gradual increase in parallel with the severity of liver fibrosis, in a stepwise manner (Supplementary Fig. 2), with a rho value of $0.548(p<0.001)$ in $\mathrm{CHB}, 0.613(p<0.001)$ in $\mathrm{NASH}$, $0.655(p<0.001)$ in $\mathrm{AIH}$, and $0.572(p<0.001)$ in PBC. Next, the correlation between LSM and the activity of liver necroinflammation was investigated. The rho values were $0.517(p<0.001)$ in $\mathrm{CHB}, 0.547(p<0.001)$ in $\mathrm{NASH}$, $0.742(p<0.001)$ in $\mathrm{AIH}$, and $0.459(p<0.001)$ in PBC respectively (Fig. $1 A \& B$ ). Such strong correlation with the degree of liver necroinflammatory activity may implicate a linearly dependent increase of LSM values to the worsening 
A

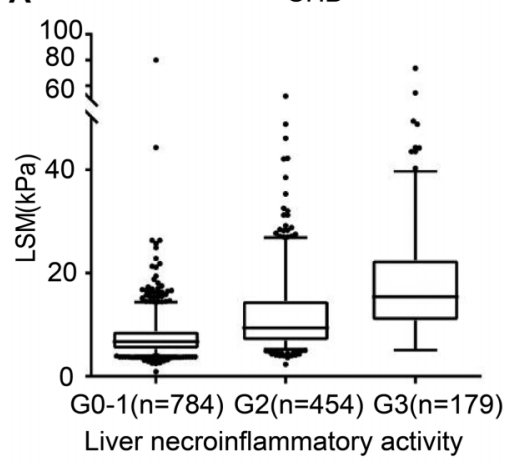

C

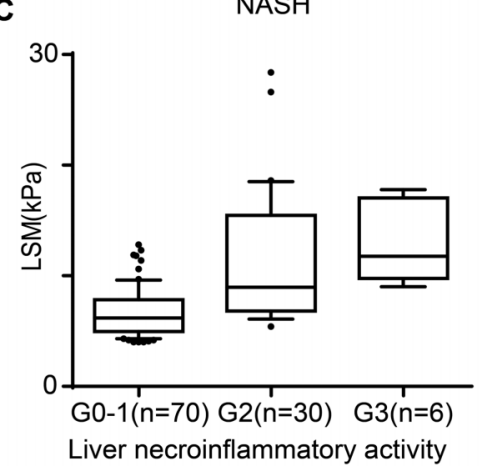

B

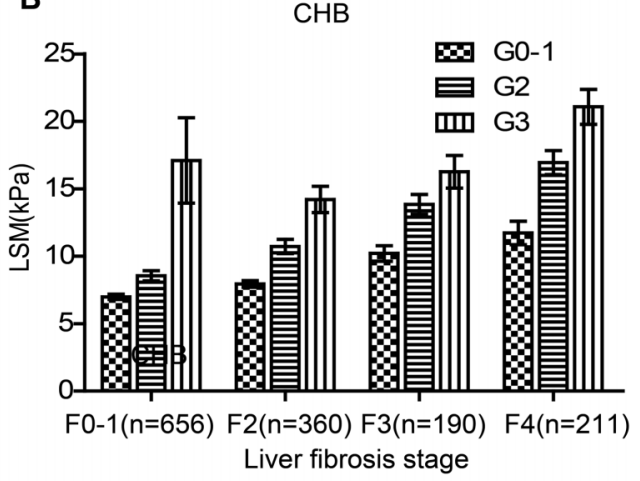

D

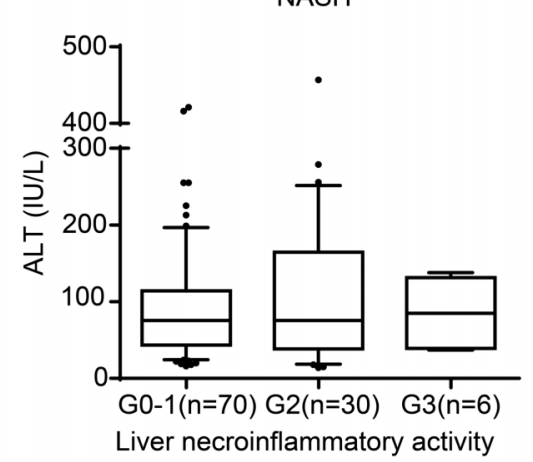

Fig. 1. The LSM value associated with liver necroinflammation. LSM value in (A) chronic hepatitis $B$ patients with different liver necroinflammatory grades and (B) the patients with same fibrosis stage. (C) LSM value and (D) alanine aminotransferase value in NASH patients with different liver necroinflammatory grades in NASH patients. Abbreviations: LSM, liver stiffness measurement; NASH, non-alcoholic steatohepatitis.

of liver necroinflammatory severity, as shown in Fig. $1 \mathrm{~A}$ for CHB patients and Fig. 1C for NASH patients. Based on the fact that the occurrence of hepatic fibrosis usually accompanied active liver inflammation, it was reasonable to suspect that in the current study, the stepwise increase of LSM with liver necroinflammation could be simply due to its direct correlation with fibrosis. In order to exclude liver fibrosis as the common responding variable for LSM eleva- tion, $\mathrm{CHB}$ patients were divided into subgroups according to their liver fibrotic scores. As shown in Fig. 1B, gradual increases of LSM with liver inflammation severity were still observed, though the severity of fibrosis in each subgroup was the same.

To further confirm that the correlation between LSM and liver necroinflammation was independent to liver fibrosis, covariance analysis was performed. It revealed that the
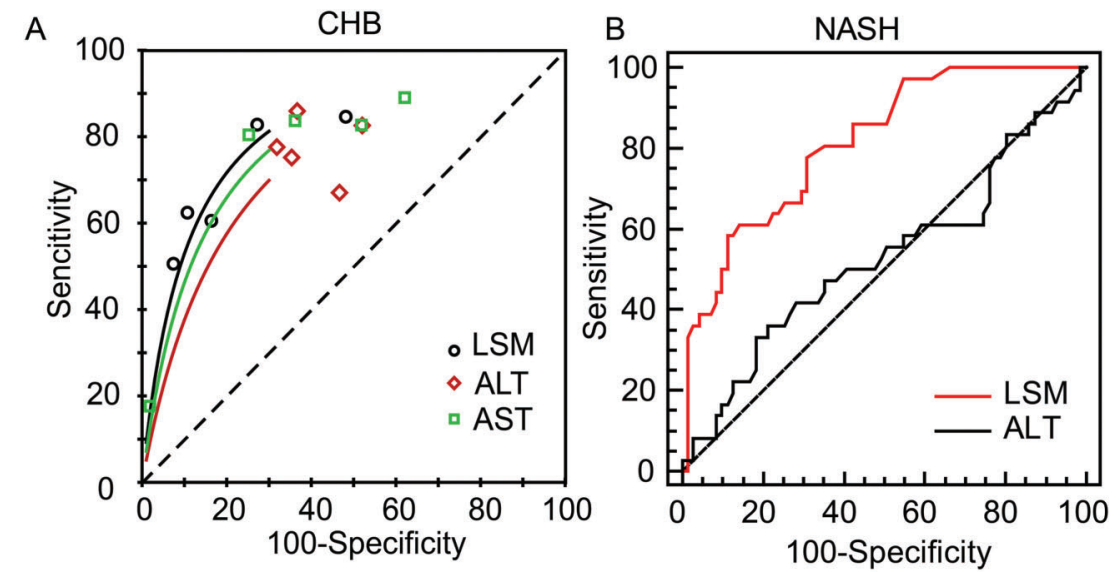

Fig. 2. Diagnostic performance of LSM for the non-invasive assessment of moderate liver necroinflammatory grade. (A) The summary receiver operating characteristic curves of LSM, alanine aminotransferase and aspartate aminotransferase in chronic hepatitis B patients. (B) Diagnostic performance of LSM and alanine aminotransferase for the non-invasive assessment of moderate liver necroinflammatory grade in NASH patients.

Abbreviations: LSM, liver stiffness measurement; NASH, non-alcoholic steatohepatitis. 
Wang L. et al: LSM predicts liver necroinflammation in CLD

Table 2. Diagnostic performance of LSM and ALT for the non-invasive assessment of liver necroinflammation

\begin{tabular}{|c|c|c|c|c|}
\hline \multirow[b]{2}{*}{ Necroinflammation } & \multicolumn{2}{|l|}{$\mathrm{CHB}, n=1417$} & \multicolumn{2}{|l|}{$\mathrm{NASH}, n=106$} \\
\hline & $\begin{array}{l}\text { G0-1 vs. G2-3 } \\
\text { (784 vs. } 633 \text { ) }\end{array}$ & $\begin{array}{l}\text { G0-2 vs. G } 3 \\
(1238 \text { vs. } 179)\end{array}$ & $\begin{array}{l}\text { G0-1 vs. G2-3 } \\
\text { (70 vs. } 36)\end{array}$ & $\begin{array}{l}\text { G0- } 1 \text { vs. G2-3 } \\
(70 \text { vs. } 36)\end{array}$ \\
\hline \multicolumn{5}{|l|}{ LSM } \\
\hline AUROC $(95 \% \mathrm{CI})$ & $0.779(0.756-0.800)$ & $0.838(0.817-0.856)$ & $0.826(0.740-0.893)$ & $0.871(0.792-0.928)$ \\
\hline Cut-off values & 10.2 & 10.4 & 8.7 & 8.9 \\
\hline $\begin{array}{l}\text { Sensitivity/ } \\
\text { specificity (\%) }\end{array}$ & $55 / 86$ & $78 / 75$ & $61 / 87$ & $100 / 78$ \\
\hline PPV/NPV (\%) & $75.8 / 70.4$ & $31.2 / 95.9$ & $71 / 81$ & $18.5 / 98.7$ \\
\hline $\begin{array}{l}\text { Positive/ } \\
\text { negative LR }\end{array}$ & $3.66 / 0.52$ & $3.13 / 0.30$ & $4.75 / 0.45$ & $3.79 / 0.21$ \\
\hline \multicolumn{5}{|l|}{ ALT } \\
\hline AUROC $(95 \% \mathrm{CI})$ & $0.694(0.669-0.718)$ & $0.676(0.651-0.701)$ & $0.521(0.422-0.619)$ & $0.538(0.438-0.635)$ \\
\hline Cut-off values & 41 & 37 & 122 & 81 \\
\hline $\begin{array}{l}\text { Sensitivity/ } \\
\text { specificity (\%) }\end{array}$ & $66 / 63$ & $78 / 47$ & $33 / 81$ & $67 / 57$ \\
\hline PPV/NPV (\%) & $59.8 / 68.7$ & $18.1 / 93.3$ & $48 / 70$ & $9 / 97$ \\
\hline $\begin{array}{c}\text { Positive/ } \\
\text { negative LR }\end{array}$ & $1.80 / 0.54$ & $1.47 / 0.47$ & $1.79 / 0.82$ & $1.55 / 0.58$ \\
\hline
\end{tabular}

ALT, alanine transaminase; AUROC, area under the ROC curve; CHB, chronic hepatitis B; LR, likelihood ratio; LSM, liver stiffness measurement; NASH, non-alcoholic steatohepatitis; NPV, negative predictive value; PPV, positive predictive value.

correlation between LSM and liver necroinflammation was independent of liver fibrosis. All these results verified that the latent capability of LSM to reflect liver necroinflammation is independent of liver fibrosis.

\section{LSM showed diagnostic potential for moderate and severe liver necroinflammation in the CHB patients}

As shown in Table 2, with cut-off value set at $10.2 \mathrm{kPa}$, LSM exhibited good diagnostic value to distinguish $\mathrm{CHB}$ patients with moderate to severe liver necroinflammation from those with only mild or without liver inflammation. Moreover, it could even identify if a CHB patient had severe liver inflammation (G3) when the best cut-off value was set as $10.4 \mathrm{kPa}$. Such diagnosis power for moderate to severe liver necroinflammation (G0-1 vs. G2-3) was further validated separately in all five independent cohorts, with AUROCs that varied from 0.780 (cohort C) to 0.829 (cohort E) (Supplementary Table 2).

By use of RevMan 5.3, the data from five independent centers were analyzed. Summary ROC curves were also plotted to evaluate the overall inflammation diagnostic value of LSM, alanine aminotransferase, and aspartate aminotransferase respectively. As shown in Fig. 2B, compared to alanine aminotransferase and aspartate aminotransferase, LSM performed statistically significant higher AUROC. LSM and aspartate aminotransferase both exhibited reliable diagnostic value in different cohorts, while alanine aminotransferase showed a large difference in five cohorts. Taken together, LSM in the CHB cohorts exhibited good diagnostic value for moderate to severe liver necroinflammation. In order to explore whether the different devices would affect the diagnostic accuracy of LSM for liver necroinflammation, a subgroup analysis was performed. The results showed that excluding cohort
C or cohort E (performed by FibroTouch) would not affect the overall diagnostic accuracy of LSM, which means different devices would not affect the diagnostic accuracy of LSM.

Considering that moderate liver necroinflammation and significant liver fibrosis were both necessary indications of anti-viral treatment in clinical use, the diagnostic value of LSM for moderate necroinflammation and/or fibrosis was also explored by a strategy mimicking meta-analysis. The results showed that with $59 \%$ (55-62\%) sensitivity, 88\% (85-91\%) specificity and $10.259(7.340,14.338)$ diagnostic odds ratio, LSM had moderate diagnostic value. The positive likelihood ratio and negative likelihood ratio were $4.9(3.8,6.4)$ and $0.47(0.43,0.52)$ respectively.

\section{Decline of LSM value was found to accompany the improvement of liver inflammatory activity in the CHB patients after receiving anti-viral treatment}

To further confirm that the value of LSM was associated with the activity of liver necroinflammation, the dynamic changes of LSM among a small longitudinal cohort of CHB patients who underwent anti-viral treatment was analyzed. As expected, after receiving entecavir treatment, alanine aminotransferase activity returned to normal in 13 weeks and was maintained at a low level thereafter. Though slower than the rapid decline of alanine aminotransferase activity, the values of LSM also dramatically declined in the first 26 weeks post-treatment initiation, and no obvious change was observed thereafter (Fig. 3). To uncover the driving factors that contributed to the rapid decline in LSM value seen in CHB patients after receiving anti-viral therapy, histopathological evaluation was conducted via liver biopsy at week 78 post-treatment initiation. In contrast to the largely unchanged fibrotic scores $(2.36 \pm$ 0.75 vs. $2.14 \pm 0.95)$, the liver micro-environmental 
necroinflammation severity grades at 78 weeks post-initiation of anti-viral treatment was significantly improved, declining from $2.79 \pm 0.89$ at baseline to $1.86 \pm 0.77(p<0.001)$. Thus, an early sharp drop of LSM post-initiation of treatment likely implicated the improvement of liver necroinflammation, same as what the rapid decline of alanine aminotransferase indicated. These results further supported our hypothesis that increased LSM values represent active liver necroinflammation in patients with chronic liver disease, at least in some cases.

\section{LSM diagnostic value for moderate and severe liver necroinflammation in the NASH population}

As shown in Table 3, alanine aminotransferase failed to display a correlation with the severity of liver necroinflammation in the NASH patient group (rho $=0.037, p=0.704$ ), which was in contrast to its strong correlations with the severity of necroinflammation (rho $=0.342, p<0.001$ ) in the $\mathrm{CHB}$ cohort. Concordant with alanine aminotransferase, direct bilirubin (rho $=0.067, p=0.495$ ), gamma-glutamy transpeptidase (rho $=0.135, p=0.169$ ), total bile acid (rho $=0.059, p=0.549$ ), alkaline phosphatase (rho $=$ $0.239, p=0.014$ ) and aspartate aminotransferase (rho = $0.194, p=0.047$ ), the currently commonly used indexes, did not exhibit a strong correlation with the severity of liver necroinflammation in the NASH patient group. Obviously, there is a great need to develop additional markers or indicators to reflect liver necroinflammation for NASH patients.

TE-based controlled attenuation parameter has become a reliable method for fatty liver diagnosis, which indicates the multiple potentials for a TE-based method to reflect different histological features of the liver. ${ }^{23}$ Meanwhile, previous studies have already revealed the liver inflammation diagnostic potential of magnetic resonance elastography, an ultrasound-based technique. ${ }^{24}$ As shown above, LSM values showed a strong correlation with the degree of liver inflammatory activity. Since LSM is also an ultrasound-based technique, it may be worthwhile to explore the potential use of TE-based LSM in the diagnosis of active liver necroinflammation in NASH patients, and if possible to utilize LSM to better score the degree of inflammatory activity. Following this idea, the ROC curves were plotted. As shown in Table 2 and Fig. 2A,

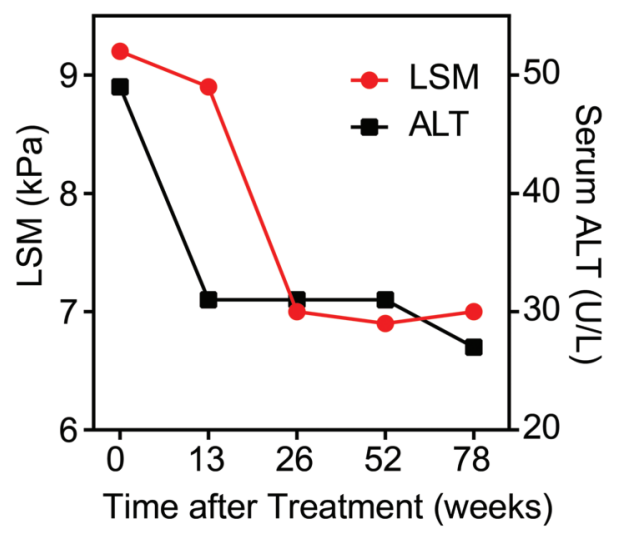

Fig. 3. In longitudinal cohort LSM and alanine aminotransferase (median tinterquartile range) level change during treatment with entecavir.

Abbreviation: LSM, liver stiffness measurement.
Wang L. et al: LSM predicts liver necroinflammation in CLD

Table 3. Correlation between liver necroinflammation and clinical characteristics

\begin{tabular}{llllll}
\hline & \multicolumn{2}{l}{$\mathrm{CHB}, \mathrm{n}=1417$} & & \multicolumn{2}{l}{ NASH, $\mathrm{n}=106$} \\
\cline { 2 - 3 } Variables & rho & $p$-value & & rho & $p$-value \\
\hline ALT (U/L) & 0.342 & $<0.001$ & & 0.037 & 0.704 \\
AST (U/L) & 0.413 & $<0.001$ & & 0.194 & 0.047 \\
TBIL & 0.106 & $<0.001$ & & -0.053 & 0.585 \\
(umol/L) & & & & \\
DBIL & 0.266 & $<0.001$ & & 0.067 & 0.495 \\
(umol/L) & & & & \\
GGT (U/L) & 0.459 & $<0.001$ & & 0.135 & 0.169 \\
ALP (U/L) & 0.272 & $<0.001$ & & 0.239 & 0.014 \\
TBA & 0.312 & $<0.001$ & & 0.059 & 0.549 \\
(umol/L) & & & & \\
LSM (kPa) & 0.517 & $<0.001$ & & 0.547 & $<0.001$ \\
Fibrosis & 0.531 & $<0.001$ & 0.541 & $<0.001$ \\
stage & & & &
\end{tabular}

Abbreviations: ALT, alanine aminotransferase; AST, aspartate aminotransferase; DBIL, direct bilirubin; ALP, alkaline phosphatase; TBA, total bile acid; GGT, gamma glutamyl transpeptidase; LSM, liver stiffness measurement.

LSM exhibited a good diagnostic value for moderate liver inflammation $(\geq G 2)$ and severe inflammation $(\geq G 3)$. In NASH patients, the AUROC curves of LSM for diagnosing moderate and severe liver inflammation were significantly higher than that of alanine aminotransferase (0.826 vs. 0.528 , $p<0.0001$ and 0.871 vs. $0.538, p=0.0011)$, respectively. The data here further confirmed the poor performance of alanine aminotransferase as a surrogate to identify liver necroinflammatory grade in NASH patients, as previously reported. ${ }^{25}$

\section{LSM could distinguish moderate and severe liver necroinflammation in the autoimmune-related liver disease cohort}

In order to further demonstrate that the diagnostic potential of LSM for liver necroinflammation was etiology-independent, the ROC curves were also plotted for AIH and PBC patients, respectively. As shown in Table 4, LSM exhibited good diagnostic performances for moderate liver inflammation ( $\geq \mathrm{G} 2)$ and severe inflammation $(\geq G 3)$ not only in the AIH patient group but also in the PBC patient group. In AIH patients, the AUROC curves of LSM for diagnosing moderate and severe liver inflammation were 0.880 and 0.907 respectively. In PBC patients, the AUROCs of LSM were 0.815 and 0.732 for diagnosing moderate and severe liver inflammation respectively. These data further confirmed the capability of LSM to discriminate liver necroinflammatory was etiologyindependent.

\section{Discussion}

Rather than consider the existence of liver inflammation as a noise factor for the accurate diagnosis of cirrhosis by TEbased LSM, here in this study, we tried to explore the potential application of TE to reflect the severity of liver necroinflammation in chronic liver diseases with different etiologies. To 
Wang L. et al: LSM predicts liver necroinflammation in CLD

Table 4. Diagnostic performance of LSM for the non-invasive assessment of liver necroinflammation

\begin{tabular}{|c|c|c|c|c|}
\hline \multirow[b]{2}{*}{ Necroinflammation } & \multicolumn{2}{|l|}{$\mathrm{AIH}, n=66$} & \multicolumn{2}{|l|}{$\mathrm{PBC}, n=77$} \\
\hline & $\begin{array}{l}0-1 \text { vs. G2-3 } \\
(11 \text { vs. } 55)\end{array}$ & $\begin{array}{l}\text { G0-2 vs. G } 3 \\
(33 \text { vs. } 33 \text { ) }\end{array}$ & $\begin{array}{l}\text { G0-1 vs. G2-3 } \\
(14 \text { vs. } 63)\end{array}$ & $\begin{array}{l}\text { G0-2 vs. G } 3 \\
(37 \text { vs. } 40)\end{array}$ \\
\hline \multicolumn{5}{|l|}{ LSM } \\
\hline AUROC $(95 \% \mathrm{CI})$ & $0.880(0.777-0.947)$ & $0.907(0.810-0.965)$ & $0.815(0.710-0.894)$ & $0.732(0.619-0.827)$ \\
\hline Cut-off values & 6.1 & 10.1 & 6.8 & 6.8 \\
\hline $\begin{array}{l}\text { Sensitivity/specificity } \\
\text { in } \%\end{array}$ & $80.00 / 90.91$ & $81.82 / 90.91$ & $71.43 / 85.71$ & $78.38 / 55.00$ \\
\hline PPV/NPV in \% & $97.8 / 47.6$ & $90.0 / 83.3$ & $95.7 / 40.0$ & $59.1 / 66.7$ \\
\hline Positive/negative LR & $8.80 / 0.22$ & $9.00 / 0.20$ & $5.00 / 0.33$ & $1.56 / 0.54$ \\
\hline
\end{tabular}

Abbreviations: AIH, auto-immune hepatitis; AUROC, area under the ROC curve; LR, likelihood ratio; LSM, liver stiffness measurement; NPV, negative predictive value; PBC, primary biliary cholangitis; PPV, positive predictive value.

provide supporting evidence to our hypothesis, the relationship between values of LSM and degree of liver inflammation was first assessed through various aspects. As expected, the aberrant elevation of LSM was consistent with the severity of liver necroinflammation seen in all chronic liver diseases, including $\mathrm{CHB}, \mathrm{NASH}$ and autoimmune-related liver diseases (autoimmune disease and PBC) populations (Fig. 1A, 1C \& Supplementary Fig. 1). Importantly, after divid-ing the large-sample sized group of CHB patients into subgroups according to each individual's fibrotic scores, the correlation between elevated LSM values and the severity of liver necroinflammation still existed in every subgroup of $\mathrm{CHB}$ patients with the same fibrotic scores. To further validate our findings, by use of the idea of meta-analysis, we verified LSM's capability to respond to inflammatory activities in five independent cohorts composed of $1417 \mathrm{CHB}$ patients in total (Fig. 3). We believe that this meta-analysis-mimicking approach is more authentic and more reliable than traditional meta-analysis due to the fact that the raw data was obtained from each research hospital.

Another important piece of evidence in support of our idea is that increased LSM could respond to active liver inflammation came from the observation of the dynamic change of LSM in CHB patients who underwent anti-viral therapy. As revealed in this longitudinal cohort, LSM decreased rapidly during the first 13-26 weeks of treatment in these CHB patients, after the recovery of liver inflammation achieved by anti-viral treatment (indicated by the decline of alanine aminotransfer-ase). As is known, the fast drop in LSM values in such a short time could not be contributed to the regression of liver fibrosis, and this was confirmed by the pathologic evaluation of inflammation activity and status of fibrotic lesions. We suggest that after the necroinflammation was controlled by anti-viral treatment, the equilibrium value thereafter might represent the true fibrotic scores of the patients.

As important histological features, necroinflammatory grade and liver fibrosis were the main indicators for the prediction of disease prognosis and evaluation of treatment benefit. ${ }^{26-28}$ The former represents ongoing liver injury and the latter stands for accumulated lesions. It has been acknowledged that in general, patients with no or mild inflammation usually have slower disease progression, ${ }^{29-31}$ while those with active liver necroinflammation display more progressive disease processes. ${ }^{32}$ In line with this, findings from numerous studies support the idea that liver inflammation drives the rise of fibrosis by accelerating hepatocyte necrosis and apoptosis. Since the presence of moderate liver necroinflammation and fibrosis was considered as the index of progressive liver disease, ${ }^{33}$ it has been recommended by major guidelines that, in clinical practice, moderate liver necroinflammation and/or moderate liver fibrosis are indicators for immediate initiation of anti-viral therapy in CHB patients ${ }^{2}$ or need of strong intervention for NASH patients. To identify those chronic liver disease patients with ongoing liver necroinflammatory damage, alanine aminotransferase level has been recommended by all major guidelines as a surrogate marker for hepatic damage in and indicator for anti-viral treatment of CHB patients. ${ }^{2,3,16}$ As is known, alanine aminotransferase might remain normal or only mildly elevated (up to $13-47 \%$ ) in $\mathrm{CHB}$ patients with active liver inflammatory activity, $8,9,34,35$ or even worse has almost no value for the diagnosis of inflammation damage among NASH patients, as previously reported ${ }^{36-38}$ and shown in the current study. Serum CK-18 was found to be elevated in the NASH population compared to the non-alcoholic fatty liver population. However, few studies have evaluated whether CK-18 could act as a non-invasive biomarker to reflect NASH-related necroinflammatory activity. ${ }^{39}$ With the impressive prevalence of non-alcoholic fatty liver disease population in China and worldwide in recent decades, there is an urgent need to develop a novel method to meet this clinical need. Currently, LSM's potential diagnostic value for liver necroinflammation deserves more attention, particularly for NASH patients.

Therefore, the potential use of LSM as a supplementary indicator for liver necroinflammation was explored in the current study. Indeed, in the current chronic liver disease cohort with etiologies including $\mathrm{CHB}, \mathrm{NASH}$ and autoimmune liver diseases, LSM exhibited certain diagnostic performance for moderate liver necroinflammation. In addition, LSM provided a potential means to survey whether anti-viral therapy had improved necroinflammation in $\mathrm{CHB}$ patients. For example, a constant decline in LSM value might implicate the efficient control of liver necroinflammation in $\mathrm{CHB}$ patients who have undergone anti-viral therapy. From this respect, LSM provides a more accurate assessment of the disease progression than we thought previously, which reflects ongoing liver necroinflammation in addition to cumulative liver damage (liver fibrosis/cirrhosis) over time. Meanwhile, the dual characteristics of LSM might be helpful to a 
clinician to decide whether or not to initiate anti-viral treatment for individuals with chronic HBV infection, rather than setting a different cut-off value to distinguish inflammation from fibrosis, or vice versa, for $\mathrm{CHB}$ patients with different alanine aminotransferase levels.

The relatively small overall number of NASH participants is also a limitation of this study. Other limitations of this study are that the chronic liver disease patients with etiologies such as hepatitis $C$ virus, alcoholic liver disease and drug-induced liver disease were not collected and analyzed.

In summary, results in this study suggest that TE-based LSM can identify moderate and severe liver inflammation with high accuracy in $\mathrm{CHB}, \mathrm{NASH}$, and autoimmune-related liver disease patients. We suggest that rather than search for a novel non-invasive biomarker, taking full advantage of the present TE-based LSM for the diagnosis of active liver necroinflammation may be more practical.

\section{Acknowledgments}

This work was supported by the National S \& T Major Project for Infectious Diseases (No.2017ZX10201201, 2017ZX10202202, 2017ZX10302201 and 2017ZX10202203), the project from Beijing Municipal Science \& Technology Commission (No.Z161100000116047), Project funded by China Postdoctoral Science Foundation (No. 2017M620544, 2018T110014) and Project funded by Shenzhen Municipal Health Commission (No. SZSM201612071).

\section{Conflict of interest}

The authors have no conflict of interests related to this publication.

\section{Author contributions}

Conceived the idea and designed the research (FL, MY, LW, $Y L)$, collected data and performed the research $(X Z, Y N, L C$, ZG, HL, GH, LW, MY, MZ, XW, YZ, SD, YZ, LC, QF, JN), analyzed the data and wrote the paper ( $L W, X W, M Y)$, and revised the paper $(F L, J Z, Y L)$.

\section{References}

[1] Asrani SK, Devarbhavi H, Eaton J, Kamath PS. Burden of liver diseases in the world. J Hepatol 2019;70:151-171. doi: 10.1016/j.jhep.2018.09.014.

[2] EASL 2017 Clinical Practice Guidelines on the management of hepatitis B virus infection. J Hepatol 2017;67:370-398. doi: 10.1016/j.jhep.2017.03.021.

[3] Terrault NA, Bzowej NH, Chang KM, Hwang JP, Jonas MM, Murad MH. AASLD guidelines for treatment of chronic hepatitis B. Hepatology 2016; 63:261-283. doi: 10.1002/hep.28156.

[4] Wang FS, Fan JG, Zhang Z, Gao B, Wang HY. The global burden of liver disease: the major impact of China. Hepatology 2014;60:2099-2108. doi: 10.1002/hep. 27406

[5] Younossi ZM, Otgonsuren M, Henry L, Venkatesan C, Mishra A, Erario M, et al. Association of nonalcoholic fatty liver disease (NAFLD) with hepatocellular carcinoma (HCC) in the United States from 2004 to 2009. Hepatology 2015;62:1723-1730. doi: 10.1002/hep.28123.

[6] Younossi ZM, Koenig AB, Abdelatif D, Fazel Y, Henry L, Wymer M. Global epidemiology of nonalcoholic fatty liver disease-Meta-analytic assessment of prevalence, incidence, and outcomes. Hepatology 2016;64:73-84. doi: 10.1002/hep. 28431

[7] Ito $T$, Ishigami $M$, Ishizu $Y$, Kuzuya $T$, Honda $T$, Hayashi $K$, et al. Utility and limitations of noninvasive fibrosis markers for predicting prognosis in biopsyproven Japanese non-alcoholic fatty liver disease patients. J Gastroentero Hepatol 2019;34:207-214. doi: 10.1111/jgh.14448.
[8] Göbel T, Erhardt A, Herwig M, Poremba C, Baldus SE, Sagir A, et al. High prevalence of significant liver fibrosis and cirrhosis in chronic hepatitis $B$ patients with normal ALT in central Europe. J Med Virol 2011;83:968-973. doi: $10.1002 / j m v .22048$

[9] Nguyen K, Pan C, Xia V, Hu J, Hu KQ. Clinical course of chronic hepatitis B (CHB) presented with normal ALT in Asian American patients. J Viral Hepat 2015;22:809-816. doi: 10.1111/jvh.12388.

[10] Kleiner DE, Makhlouf HR. Histology of nonalcoholic fatty liver disease and nonalcoholic steatohepatitis in adults and children. Clin Liver Dis 2016;20: 293-312. doi: 10.1016/j.cld.2015.10.011.

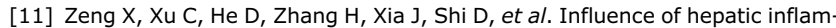
mation on fibroscan findings in diagnosing fibrosis in patients with chronic hepatitis B. Ultrasound Med Biol 2015;41:1538-1544. doi: 10.1016/j.ultrasmedbio.2015.01.011.

[12] Boursier J, Vergniol J, Guillet A, Hiriart JB, Lannes A, Le Bail B, et al. Diagnostic accuracy and prognostic significance of blood fibrosis tests and liver stiffness measurement by FibroScan in non-alcoholic fatty liver disease. J Hepatol 2016;65:570-578. doi: 10.1016/j.jhep.2016.04.023.

[13] Yan LB, Zhu X, Bai L, Liang LB, Chen EQ, Du LY, et al. Impact of mild to moderate elevations of alanine aminotransferase on liver stiffness measurement in chronic hepatitis B patients during antiviral therapy. Hepatol Res 2013;43:185-191. doi: 10.1111/j.1872-034X.2012.01068.X.

[14] Kong Y, Sun Y, Zhou J, Wu X, Chen Y, Piao H, et al. Early steep decline of liver stiffness predicts histological reversal of fibrosis in chronic hepatitis $B$ patients treated with entecavir. J Viral Hepat 2019;26:576-585. doi: 10. $1111 /$ jvh. 13058 .

[15] Dong XQ, Wu Z, Li J, Wang GQ, Zhao H. Declining in liver stiffness cannot indicate fibrosis regression in patients with chronic hepatitis B: A 78-week prospective study. J Gastroenterol Hepatol 2019;34:755-763. doi: 10. $1111 /$ jgh. 14498.

[16] Liaw YF, Kao JH, Piratvisuth T, Chan HL, Chien RN, Liu CJ, et al. Asian-Pacific consensus statement on the management of chronic hepatitis B: a 2012 update. Hepatol Int 2012;6:531-561. doi: 10.1007/s12072-012-9365-4.

[17] Ratziu V, Bellentani S, Cortez-Pinto H, Day C, Marchesini G. A position statement on NAFLD/NASH based on the EASL 2009 special conference. J Hepatol 2010;53:372-384. doi: 10.1016/j.jhep.2010.04.008

[18] Yao M, Wang L, Leung PSC, Li Y, Liu S, Wang L, et al. The clinical significance of GP73 in immunologically mediated chronic liver diseases: Experimental data and literature review. Clin Rev Allergy Immunol 2018;54:282-294 doi: 10.1007/s12016-017-8655-y.

[19] Hou J, Wang G, Wang F, Cheng J, Ren H, Zhuang $\mathrm{H}$, et al. Guideline of prevention and treatment for chronic hepatitis B (2015 update). J Clin Transl Hepatol 2017;5:297-318. doi: 10.14218/JCTH.2016.00019.

[20] Scheuer P. Primary biliary cirrhosis. Proc R Soc Med 1967;60:1257-1260. doi: $10.1177 / 003591576706001205$.

[21] Brunt EM, Janney CG, Di Bisceglie AM, Neuschwander-Tetri BA, Bacon BR. Nonalcoholic steatohepatitis: a proposal for grading and staging the histological lesions. Am J Gastroenterol 1999;94:2467-2474. doi: 10.1111/j.15720241.1999.01377.x.

[22] Herrmann E, de Lédinghen V, Cassinotto C, Chu WC, Leung VY, Ferraioli G, et al. Assessment of biopsy-proven liver fibrosis by two-dimensional shea wave elastography: An individual patient data-based meta-analysis. Hepatology 2018;67:260-272. doi: 10.1002/hep.29179.

[23] Eddowes PJ, Sasso M, Allison M, Tsochatzis E, Anstee QM, Sheridan D, et al. Accuracy of fibroscan controlled attenuation parameter and liver stiffness measurement in assessing steatosis and fibrosis in patients with nonalcoholic fatty liver disease. Gastroenterology 2019;156:1717-1730. doi: 10.1053/j.gastro.2019.01.042.

[24] Yin M, Glaser KJ, Manduca A, Mounajjed T, Malhi H, Simonetto DA, et al. Distinguishing between hepatic inflammation and fibrosis with MR elastography. Radiology 2017;284:694-705. doi: 10.1148/radiol.2017160622.

[25] Younossi ZM, Loomba R, Anstee QM, Rinella ME, Bugianesi E, Marchesini G, et al. Diagnostic modalities for nonalcoholic fatty liver disease, nonalcoholic steatohepatitis, and associated fibrosis. Hepatology 2018;68:349-360. doi: 10.1002/hep.29721.

[26] Sanyal AJ, Chalasani N, Kowdley KV, McCullough A, Diehl AM, Bass NM, et al. Pioglitazone, vitamin $\mathrm{E}$, or placebo for nonalcoholic steatohepatitis. N Engl J Med 2010;362:1675-1685. doi: 10.1056/NEJMoa0907929.

[27] Neuschwander-Tetri BA, Clark JM, Bass NM, Van Natta ML, Unalp-Arida A, Tonascia J, et al. Clinical, laboratory and histological associations in adults with nonalcoholic fatty liver disease. Hepatology 2010;52:913-924. doi: 10 . 1002/hep. 23784

[28] Younossi ZM, Stepanova M, Rafiq N, Makhlouf H, Younoszai Z, Agrawal R, et al. Pathologic criteria for nonalcoholic steatohepatitis: interprotocol agreement and ability to predict liver-related mortality. Hepatology 2011 53:1874-1882. doi: 10.1002/hep.24268

[29] McMahon BJ. The natural history of chronic hepatitis B virus infection. Semin Liver Dis 2004;24 Suppl 1:17-21. doi: 10.1055/s-2004-828674. 
Wang L. et al: LSM predicts liver necroinflammation in CLD

[30] Hoofnagle JH, Doo E, Liang TJ, Fleischer R, Lok AS. Management of hepatitis B: summary of a clinical research workshop. Hepatology 2007; 45:1056-1075. doi: 10.1002/hep.21627.

[31] Fattovich G. Natural history and prognosis of hepatitis B. Semin Liver Dis 2003;23:47-58. doi: 10.1055/s-2003-37590.

[32] Czaja AJ. Hepatic inflammation and progressive liver fibrosis in chronic liver disease. World J Gastroenterol 2014;20:2515-2532. doi: 10.3748/wjg.v20. i10.2515.

[33] Asselah T, Boyer N, Guimont MC, Cazals-Hatem D, Tubach F, Nahon K, et al. Liver fibrosis is not associated with steatosis but with necroinflammation in French patients with chronic hepatitis C. Gut 2003;52:1638-1643. doi: 10. 1136/gut.52.11.1638

[34] Sarin SK, Kumar M. Should chronic HBV infected patients with normal ALT treated: debate. Hepatol Int 2008;2:179-184. doi: 10.1007/s12072-0089065-2.

[35] Lesmana CR, Gani RA, Hasan I, Simadibrata M, Sulaiman AS, Pakasi LS, et al. Significant hepatic histopathology in chronic hepatitis B patients with serum
ALT less than twice ULN and high HBV-DNA levels in Indonesia. J Dig Dis 2011;12:476-480. doi: 10.1111/j.1751-2980.2011.00540.x.

[36] Browning JD, Szczepaniak LS, Dobbins R, Nuremberg P, Horton JD, Cohen JC, et al. Prevalence of hepatic steatosis in an urban population in the United States: impact of ethnicity. Hepatology 2004;40:1387-1395. doi: 10.1002/hep. 20466

[37] Sorrentino P, Tarantino G, Conca P, Perrella A, Terracciano ML, Vecchione R, et al. Silent non-alcoholic fatty liver disease-a clinical-histological study. J Hepatol 2004;41:751-757. doi: 10.1016/j.jhep.2004.07.010.

[38] Mofrad P, Contos MJ, Haque M, Sargeant C, Fisher RA, Luketic VA, et al. Clinical and histologic spectrum of nonalcoholic fatty liver disease associated with normal ALT values. Hepatology 2003;37:1286-1292. doi: 10.1053/jhep. 2003.50229.

[39] Feldstein AE, Wieckowska A, Lopez AR, Liu YC, Zein NN, McCullough AJ. Cytokeratin-18 fragment levels as noninvasive biomarkers for nonalcoholic steatohepatitis: a multicenter validation study. Hepatology 2009; 50:1072-1078. doi: 10.1002/hep.23050. 